\title{
RESISTIR DESDE EL ESTADO. \\ LA CONSTRUCCIÓN DE ESTRATEGIAS “DESDE ABAJO" FRENTE A LOS MODOS DE GESTIÓN DE LAS POLÍTICAS HACIA LA ESCUELA SECUNDARIA OBLIGATORIA DE CAMBIEMOS
}

\author{
Maida Diyarian* \\ Universidad de Buenos Aires, Argentina \\ mdiyarian@gmail.com \\ Inés Rodriguez Moyano* \\ Universidad de Buenos Aires, Argentina \\ inesrmoyano@gmail.com
}

Recibido: 11/08/2020 - Aceptado: 6/01/2021

\section{Resumen}

Este artículo analiza los modos de gestión que acompañan a los procesos de implementación de las políticas dirigidas a garantizar la obligatoriedad del nivel secundario en el marco de la restauración conservadora. A partir de un estudio de corte cualitativo, el presente trabajo analiza las miradas de los referentes de la gestión intermedia sobre la orientación de las políticas educativas durante el periodo 2015 - 2018 en la Provincia de Buenos Aires, así como las estrategias de resistencias a escala local para la ampliación del derecho a la educación.

Palabras clave: Políticas educativas - Restauración conservadora - Resistencias - Nivel secundario - Provincia de Buenos Aires.

RESISTING FROM THE STATE.

\section{STRATEGIES "FROM BELOW" FACING THE POLICES MANAGEMENT TOWARDS CAMBIEMOS MANDATORY HIGH SCHOOL ATTENDANCE}

\begin{abstract}
This article analyzes the systems of government aimed at guaranteeing the mandatory high school at the local level in the context of conservative restoration. Based on a qualitative study, the

\footnotetext{
* Licenciada en Sociología (UBA) y Maestranda en Ciencias Sociales con Orientación en Educación de FLACSO. Ha sido docente de Sociología de la Educación en la Facultad de Ciencias Sociales de la UBA y en el Instituto de Educación de la Universidad Nacional de Hurlingham. Actualmente se desempeña como Investigadora del Proyecto UBACYT " Políticas públicas y derecho a la educación en la Argentina del siglo XXI. Análisis sobre las trayectorias de las políticas educativas en un país federal. Dir. Myriam Feldfeber, sede Instituto de Investigaciones en Ciencias de la Educación-UBA. Ha realizado trabajos de investigación en el campo de políticas públicas y desigualdades sociales y escolares.

* Socióloga y Magister en Investigación en Ciencias Sociales (UBA). Doctoranda en Ciencias Sociales por la UBA y Profesora adjunta de la materia Sociología de Educación en la Carrera de Sociología (FSOC-UBA). Profesora de posgrado en la Maestría en Educación Virtual de la Universidad Nacional de Quilmes a cargo del Seminario de "Educación y Sociedad".
} 
REVISTA DE LA ESCUELA DE CIENCIAS DE LA EdUCACIÓN, AÑO 17, NRO. 16, VOL. 2, JULIO A DICIEMBRE dE 2021. PÁGINAS 57-67. ISSN 2362-3349 (EN LínEA). RESISTIR DESDE EL ESTADO. LA CONSTRUCCIÓN DE ESTRATEGIAS "DESDE ABAJO” FRENTE A LOS MODOS DE GESTIÓN DE LAS POLITICAS HACIA LA ESCUELA SECUNDARIA OBLIGATORIA DE CAMBIEMOS. MAIDA DIYARIAN. INÉS RODRIGUEZ MOYANO.

present work analyzes the views of the referents of intermediate management on the orientation of educational policies during 2015 - 2018 in Buenos Aires as well as the strategies of resistance at a local scale to expanding the right to education.

Keywords: Educational policies - Conservative restoration - Resistance - High school - Buenos Aires.

\section{Introducción}

Desde la última década del siglo XX, muchos países de América Latina han incorporado en sus regulaciones la meta de garantizar la obligatoriedad de la educación secundaria para hacer frente a las limitaciones no sólo en el acceso sino en la permanencia y el egreso de los jóvenes en el sistema educativo.

En Argentina, la Ley de Educación Nacional sancionada en el año 2006 extendía la obligatoriedad escolar hasta la finalización del nivel de la educación secundaria, previendo para su cumplimiento el desarrollo de "alternativas institucionales, pedagógicas y de promoción de derechos (...) mediante acciones que permitan alcanzar resultados de calidad equivalente en todo el país y en todas las situaciones sociales" (art 16, LEN). En el marco de un sistema educativo federal como el argentino - en el que los servicios educativos de todos los niveles, a excepción del universitario, se han transferido a las provincias- el análisis de las políticas educativas en general y de aquellas para garantizar la obligatoriedad de la educación secundaria en particular, supone atender a la articulación entre los distintos niveles de gestión (nacional, provincial, municipal) y a la complejidad que suma este factor al despliegue de las políticas (Feldfeber, 2011). En este contexto, la escala territorial interviene de forma específica en la orientación que adquiere la política educativa, constituyendo una dimensión relevante en el análisis de las políticas públicas. Diversos estudios han marcado cómo la transformación de la matriz socio-política estadocéntrica y la estructuración de una nueva matriz sobre la base de los procesos de privatización, descentralización y focalización que caracterizaron a las formas de intervención estatal en la Argentina durante los años '90, promovieron la configuración de nuevas lógicas de intervención donde la territorialidad y la sectorialidad adquieren centralidad, al emerger como instancias y espacios estratégicos de implementación de políticas y programas sociales (Paura y Zibecchi, 2013).

En la provincia de Buenos Aires, la preocupación por la democratización de la gestión escolar ha sido una cuestión central en la agenda política de las autoridades provinciales durante el periodo kirchnerista (2003-2015). La culminación del proceso de transferencia de servicios educativos a las jurisdicciones que tuvo lugar en la década de los '90, implicó para dicha provincia una tendencia paralela hacia la recentralización de la toma de decisiones en la instancia de la Dirección General de Cultura y Educación, y un recorte de las atribuciones de los órganos colegiados y de la administración distrital ${ }^{1}$. Con el propósito de superar las limitaciones de la centralización, desde el 2003 las autoridades provinciales buscaron desconcentrar la administración del sistema como estrategia para mejorar la gobernabilidad y el trabajo en el campo pedagógico (Agoff et. al. 2012). En esta dirección, se desplegaron diversas acciones que resultarían centrales para comprender el alcance de las políticas orientadas a garantizar la obligatoriedad del nivel secundario, definidas por la Ley Nacional de Educación (2006) y la Ley de Educación Provincial Nro. 13.688 (2007). Entre los instrumentos dispuestos a nivel organizativo-institucional que intentaron revertir el legado de los años '90, valen destacar la introducción de la regionalización como una nueva institucionalidad que se agrega al complejo entramado decisional, la redefinición del sistema de inspección, la reactivación de los Consejos Escolares y la creación de las Unidades Educativas de Gestión Distrital (UEGD) ((Agoff et. al. 2012).). La reforma de los niveles intermedios de la gestión apuntó a fortalecer la instancia distrital de planificación, gestión y seguimiento educativo conjunto, los/as inspectores/as pasaron a ocupar una posición privilegiada en la compleja trama que orienta la gobernabilidad del sistema a nivel local (íbid), otorgándoles ciertos márgenes de autonomía en las decisiones vinculadas al territorio de acción inmediato.

Con la asunción de Cambiemos en diciembre de 2015, asistimos a un cambio en las orientaciones y en los modos de construcción de las políticas públicas de la etapa precedente en el que se observa, como mostraremos en este artículo, una reversión de los procesos que tendían a la ampliación de la democratización en términos de la participación de los diferentes actores territoriales en la planificación y toma de decisiones sobre las políticas. En su lugar se evidenció una tendencia hacia la burocratización y recentralización del poder político en la cúspide de la estructura de gobierno y el consecuente debilitamiento del poder de los niveles intermedios de la gestión, que había comenzado a configurarse y fortalecerse durante los gobiernos precedentes. Asimismo, estos procesos tienden a generar dinámicas hacia la

\footnotetext{
${ }^{1}$ La reforma parcial de la Constitución de la Provincia de 1934 y la Ley de Educación Provincial Nro. 11612 de 1994 establecen un recorte en las funciones del Consejo General de Cultura y Educación (el cual es desplazado al lugar de asesoramiento reservándose la "dirección facultativa y administrativa" del sistema a la Dirección General) y de los Consejos Escolares Distritales, redimensionando el peso otorgado a la Dirección General, es decir, a la instancia unipersonal y central de conducción (Giovine, 2012). Si bien la Ley de Educación Provincial de 1994 reconocía la existencia de un espacio regional con miras a la posibilidad de encarar procesos de descentralización, el desarrollo de nuevas instancias operativas dependientes del nivel central establecidas para actuar directamente en el territorio y la concomitante limitación de las atribuciones de los órganos de gobierno locales, daban lugar a una estructura de gobierno altamente centralizada (Agoff et al., 2012).
} 
atomización y fragmentación del sistema en el marco de una agenda pública en la que la dimensión pedagógica como eje estructurador en la definición de las políticas educativas pierde centralidad.

A partir de un estudio de corte cualitativo, el presente trabajo analiza los modos de gestión que acompañan a los procesos de implementación de las políticas dirigidas a garantizar la obligatoriedad del nivel secundario en el marco de la restauración conservadora a escala local. Por un lado, interesa analizar la arquitectura institucional atendiendo a las jerarquías, funciones, atribuciones y roles que el organigrama asigna a las distintas áreas que intervienen en el diseño e implementación de las políticas dirigidas a la educación secundaria de la provincia. Por otro, indagar sobre el tipo de comunicación, canales de articulación y participación que se habilitan y promueven entre los distintos sectores y actores del sistema. Finalmente, se analizan las dinámicas de resistencia al cambio en la construcción de las políticas "desde abajo" que arrojan pistas relevantes para abrir la "caja negra" de la implementación y desentrañar los mecanismos que vehiculizan u obturan los procesos de democratización escolar.

Estos interrogantes recuperan el debate vigente en el campo académico acerca del carácter complejo, multidimensional y dinámico de las políticas, que señala la relevancia de captar la multiplicidad de actores, intereses, recursos y contextos que intervienen en la construcción e implementación de las políticas públicas. Desde esta perspectiva, adoptamos el enfoque propuesto por Stephen Ball (2002) y consideramos a las políticas en su trayectoria, es decir, en los distintos contextos que intervienen en su formulación e implementación, en los que se ponen en juego relaciones de fuerza por la imposición de sentidos. Desde aquí se entiende que los efectos de la política no son sólo el mero cumplimiento de los objetivos explicitados en éstas, sino el resultado de una trayectoria en la que intervienen diversos actores desde distintas posiciones. Siguiendo al autor, concebimos a las políticas públicas como fenómenos complejos, contradictorios y dinámicos; como textos y como discursos. Como textos, en tanto expresiones normativas codificadas de modo complejo - es decir, formuladas como resultado de relaciones de fuerza entre diversos actores - y decodificadas también de modo complejo, en función de las interpretaciones y sentidos que los actores ponen en juego en los diversos contextos locales, de acuerdo a sus posiciones, intereses, experiencias y recursos. El análisis de las políticas como textos, propuesto por Ball, implica, por lo tanto, considerar las disputas y relaciones de poder que subyacen a los procesos de diseño e implementación. A su vez, señala la relevancia de mirar no sólo las instancias de formulación y diseño enmarcadas en los niveles centrales de la gestión, sino también los modos en que los niveles intermedios de la administración e incluso las propias instituciones y docentes interpretan dichos lineamientos y en qué medida producen o no cambios en las prácticas.

Los análisis que aquí presentamos se basan en resultados de una investigación realizada desde el Instituto de Educación de la Universidad Nacional de Hurlingham, que recupera el punto de vista de los actores del nivel intermedio de la gestión, específicamente de los Inspectores Distritales y de Nivel Secundario, en tanto ocupan una posición privilegiada para analizar los procesos que acompañan la implementación de las políticas dirigidas a la educación secundaria, al actuar como "enlace" o "puente" entre aquellos que planifican y diseñan las políticas educativas y las instituciones que deben ponerlas en práctica. Asimismo, se relevaron normativas y documentos referidos al organigrama de la Dirección General de Cultura y Educación y a las líneas de acción para la educación secundaria atendiendo a los procesos de recontextualización que sobre estas orientaciones se producen a nivel local en el territorio.

El trabajo de campo se llevó a cabo en el año 2018. Se realizaron entrevistas semi-estructuradas a 11 actores que en distintos niveles participan del gobierno del sistema. Se entrevistó a dos Directores de la Dirección Nacional de Políticas Socioeducativas del período kirchnerista; a una Referente Regional, a una Asesora Pedagógica y a una Referente Distrital del área de Políticas Socioeducativas de la gestión Cambiemos; a una Jefa de Inspección Distrital del período kirchnerista y a otras dos del gobierno de Cambiemos (una de las cuales había sido Inspectora de educación secundaria entre 2007 y 2015 en otro distrito de la provincia); a dos Inspectoras de nivel secundario de la gestión Cambiemos (una de las cuales se había desempeñado como Inspectora de educación secundaria entre 2007 y 2015 en otro distrito de la provincia) ${ }^{2}$ y a un Inspector de nivel que ocupó el cargo en el distrito en ambas gestiones de gobierno.

\section{Hacia la desarticulación de las instancias de participación colectiva y la concentración del control a partir de la gestión de gobierno de Cambiemos}

Como mencionamos en la introducción, a partir del 2015 se observa una reversión de los procesos que tendían a la ampliación de la democratización en términos de la participación de los diferentes actores territoriales en la planificación y toma de decisiones sobre las políticas iniciados desde 2005. En su lugar se observa una tendencia hacia la recentralización del poder político en la cúspide de la estructura de gobierno y el consecuente debilitamiento del poder de los niveles intermedios de la gestión, que había comenzado a gestarse y fortalecerse durante los gobiernos precedentes (Rodríguez Moyano y Diyarian, 2020). Por un lado, se introducen modificaciones en el organigrama que afectan la función y jerarquía de la Dirección encargada de coordinar las políticas educativas del nivel secundario. A partir de la Resolución de firma conjunta E GDEBA-DGCyE Nro. 2412 del 29 de diciembre de 2017, las Direcciones de nivel y las Direcciones de modalidad (encargadas de coordinar las políticas de cada uno de los niveles y modalidades

${ }^{2}$ La trayectoria como Inspectora de Educación Secundaria durante el período kirchnerista en otro de los distritos de la provincia de Buenos Aires le permite tanto a la Inspectora Distrital como a la de Nivel Secundario que se desempeñan durante la gestión de Cambiemos, emitir opiniones y comparaciones entre ambos períodos. 
REVISTA DE LA ESCUELA DE CIENCIAS DE LA EdUCACIÓN, AÑO 17, NRO. 16, VOL. 2, JULIO A DICIEMBRE dE 2021. PÁGINAS 57-67. ISSN 2362-3349 (EN LínEA). RESISTIR DESDE EL ESTADO. LA CONSTRUCCIÓN DE ESTRATEGIAS "DESDE ABAJO" FRENTE A LOS MODOS DE GESTIÓN DE LAS POLITICAS HACIA LA ESCUELA SECUNDARIA OBLIGATORIA DE CAMBIEMOS. MAIDA DIYARIAN. INÉS RODRIGUEZ MOYANO.

del sistema) pasan de ser "Direcciones Provinciales" a ser "Direcciones" simples, perdiendo su carácter provincial.

Según nos comentan algunos inspectores del distrito, estas transformaciones implican una desjerarquización de su rango y alcance y, por esta vía, una limitación de sus atribuciones para intervenir en la orientación de las políticas. En este nuevo esquema, la toma de decisiones se recentraliza y concentra en la Subsecretaría de Educación, mientras que aquellas Direcciones pierden poder político convirtiéndose en áreas de asesoría técnico-pedagógica para la Subsecretaría, sin capacidad de intervenir con carácter vinculante y márgenes de autonomía en la orientación de la política educativa:

La diferencia en la baja del escalafón en que las Direcciones de nivel y modalidad dejaron de ser Direcciones Provinciales para pasar a ser Direcciones a secas es el nivel de decisores que tienen los directores. Es decir, fundamentalmente a la hora de emitir disposiciones y circulares. En realidad, tanto las disposiciones como las circulares y las comunicaciones conjuntas, saben ser del orden técnico, del andamiaje de la interpretación o de la interpelación de resoluciones o de la misma ley, es decir, son los documentos que le dan estructura a las resoluciones que ya están en marcha. Antiguamente, hasta hace poco, cuando las Direcciones eran provinciales, los directores de nivel y modalidad, tomaban decisiones desde el lugar de la autonomía porque el alcance de esas decisiones era provincial. Ahora no. Ahora funcionan simplemente como el lugar de una asesoría técnico-pedagógica para la Subsecretaría de Educación. Todos los actos dispositivos, resolutivos, comunicaciones y disposiciones surgen de la Subsecretaría de Educación. La Subsecretaría es la que ha concentrado en este último tiempo todo el poder de decisión de la Dirección General de Escuelas. Las Direcciones de nivel y modalidad simplemente están funcionando como emisoras de criterios pedagógicos, por decirlo de algún modo. Y ya no tienen más ni voz ni voto, tampoco dentro de lo que era la Jefatura de Gabinete. Antes eran convocados por la Jefatura de Gabinete dentro de la Dirección General de Escuelas, y eran convocados al Consejo General de Educación dentro de la Provincia de Buenos Aires, para dar asesoría, para argumentar, defender, la implementación de alguna política pública. Bueno, hoy ya no. Esa es la gran diferencia. Es el recorte en el nivel de la democratización de la Dirección General de Escuelas para concentrar poder en la Subsecretaría (Inspectora de nivel secundario, gestión Cambiemos).

El debilitamiento del poder político de la Dirección puede leerse juntamente con la política de continuidad de los equipos intermedios de la gestión que viene caracterizando a la cartera educativa provincial del gobierno de Cambiemos (Suasnabar et. al. 2017). Tal como advierten algunos estudios sobre el tema, la gestión de Cambiemos favoreció la permanencia de los estratos medios de la burocracia educativa (asesores, equipos técnicos). De hecho, en el caso de la Educación Secundaria, se observa una continuidad no sólo de los equipos técnicos sino también de la actual directora, quien se había desempeñado como consultora del Equipo Pedagógico de la Dirección Provincial de Educación Secundaria en la gestión previa. Sin embargo, la permanencia de equipos de la gestión kirchnerista esgrimida bajo el argumento de la "neutralidad política"- según la cual la adscripción partidaria del agente pasa a segundo plano si tiene algo para ofrecer a la gestión (Suasnabar et. al. 2017) - corre paralela al cercenamiento de su poder político.

Desde la mirada de los inspectores de nivel, la nueva institucionalidad implica un quiebre del esquema horizontal de gestión que se había comenzado a configurar en la etapa previa dando lugar a una nueva modalidad que es percibida como "totalmente arbitraria" y con nula articulación entre las distintas dependencias de gobierno. En efecto, la eliminación de la Dirección de Gestión Educativa en el actual organigrama expresa con claridad la intencionalidad de debilitar las institucionalidades encargadas de diseñar agendas de gestión que involucren estrategias específicas para llevar las políticas a los territorios y traccionarlas en la diversidad situada de los contextos de la provincia de Buenos Aires, atendiendo a los requerimientos de cada modalidad y de cada nivel. Asimismo, la eliminación o limitación de los equipos de formación específica que trabajaban en dicha Dirección de Gestión Educativa puede leerse en vinculación con el debilitamiento de la agenda pedagógica de los inspectores y el consecuente fortalecimiento de las tareas burocráticas de control y de rendición de cuentas.

En el actual contexto, desde la mirada de los actores territoriales se percibe que las decisiones en materia de política educativa se toman de forma unilateral desde la Subsecretaría a través de resoluciones internas que ignoran la autoridad de otras áreas responsables de las políticas, promoviendo la fragmentación de las áreas de gobierno:

La comunicación es vía Inspección General. Es Subsecretaría, Inspección General. No pasa más por los niveles. No pasa más por los niveles... está totalmente atomizada y fragmentada la comunicación. Hay comunicaciones que recibimos nosotros los inspectores de Inspección General que involucran el puesto de trabajo de los docentes, y sin embargo la Secretaría de Asuntos Docentes, no reciben esa comunicación (Inspectora de nivel secundario, gestión Cambiemos).

En el marco de esta nueva modalidad de gestión, la comunicación tiende a restituir el carácter verticalista del mando, anulando y diluyendo los canales de articulación preexistentes entre los distintos sectores y actores del sistema. En Hurlingham, los relatos de los inspectores dan cuenta del modo en que 
REVISTA DE LA ESCUELA DE CIENCIAS DE LA EdUCACIÓN, AÑO 17, NRO. 16, VOL. 2, JULIO A DICIEMBRE DE 2021. PÁGINAS 57-67. ISSN 2362-3349 (EN LínEA). RESISTIR DESDE EL ESTADO. LA CONSTRUCCIÓN DE ESTRATEGIAS "DESDE ABAJO” FRENTE A LOS MODOS DE GESTIÓN DE LAS POLITICAS HACIA LA ESCUELA SECUNDARIA OBLIGATORIA DE CAMBIEMOS. MAIDA DIYARIAN. INÉS RODRIGUEZ MOYANO.

los canales de comunicación entre el territorio y los niveles regionales y centrales se basan en una cadena burocrática de autorizaciones que a la vez que refuerza las sanciones sobre los sujetos, deslegitima la autoridad de los agentes para resolver los problemas en el territorio, dilatando los tiempos de resolución de los conflictos:

Eh, hoy por hoy, vos no podés tener contacto con el jefe Regional sin pedir entrevista a través de tu jefe Distrital. Es decir, porque somos sancionados... si, si, somos sancionados. Lo que nos dijo la semana pasada, el jefe de Regional, es que ante determinadas cuestiones que pedían implementar, los que somos más viejos y entendemos más de los vericuetos legales, fundamentalmente de legal y técnica levantamos la mano, fue así lo que nos dijo textual: "yo les voy a decir lo mismo que me dicen en el nivel central: al que le gusta bien, y al que no, se puede ir. Tienen dos opciones: el que está en condiciones se jubila, y si no, está el retiro voluntario" (Inspectora de nivel secundario, gestión Cambiemos).

Desde la mirada de los actores territoriales, se trata de medidas tendientes a atomizar y fragmentar el sistema en el marco de una agenda pública en la que la dimensión pedagógica como eje estructurador en la definición de las políticas educativas pierde centralidad. En sintonía con el perfil "ceocrático" de la cúpula de los funcionarios de la gestión macrista (Canelo y Castellani, 2016), en el sector educativo se visibiliza una clara tendencia hacia el fortalecimiento de la lógica tecnocrática y focalizada en la orientación de las políticas, más preocupada por cuantificar y medir resultados que por brindar recursos y articular estrategias comunes orientadas a reducir las brechas sociales y educativas.

No hay líneas para efectivizar el derecho a la educación desde el lugar del aprendizaje, desde el lugar de las enseñanzas. En eso no hay líneas de acción. Las líneas de acción de ellos son monitoreo. Es decir, y resultadistas. Ellos quieren que los pibes, por ejemplo, i ¿anoche tuve que completar una planilla de monitoreo que nos pidieron desde jefatura distrital, una planilla de monitoreo con tres renglones?! ¡¿Qué monitoreo puedo hacer yo del avance de las enseñanzas, en los nudos de la enseñanza o en los nudos del aprendizaje con tres renglones?!?! ¡¡Si te piden eso en tres renglones...te mandan un Excel!! Te mandan un Excel, porque es un Excel, donde en tres renglones te hacen hacer esto: ¿los resultados del Aprender? Tomas los resultados del Aprender, tomas el resultado del primer trimestre de calificaciones, si están mejor o peor (comparar), y en tres renglones decir cuáles son tus propuestas para la mejora. Ellos quieren resultados. No les importa qué es lo que hacemos nosotros en el medio, qué se les ocurre a los inspectores hacer en el medio. Después ellos ahora están muy obsesionados por el monitoreo de la asistencia. ¿Entonces tienen el programa Asistiré, tienen el programa pirulo... tienen el programa Alumnos, me entendés? Donde los directores tienen que ir cargando la asistencia en línea. En realidad, no es que estén preocupados por la asistencia: ellos quieren medir la asistencia para medir cuántas secciones cierran a fin de año (Inspectora de nivel secundario, gestión Cambiemos).

Los cambios orientados a la recentralización del poder político en la Subsecretaría de Educación se articulan con otros mecanismos tendientes al debilitamiento del poder de los niveles intermedios de la gestión. Tal es el caso del programa "ABC padres" que depende directamente de aquella Subsecretaría, y que consiste en la organización de encuentros entre las máximas autoridades de la Dirección General de Cultura y Educación y las familias "con el objetivo de relevar sus necesidades y demandas"3.

Al igual que el "timbreo" utilizado en la campaña electoral del macrismo, se trata de una estrategia de vinculación "uno a uno" que escenifica un contacto espontáneo y directo entre los funcionarios y los padres. Siguiendo a Natanson (2018), entendemos que estos mecanismos abonan a un proceso de construcción política donde el Estado establece un tipo de vínculo con la sociedad que desplaza el eje del ciudadano al vecino reduciendo la política a un problema individual y coyuntural: "el mensaje es que son los problemas inmediatos y cotidianos los que realmente importan, los que el político (...) viene a escuchar" (Natanson, 2018: p.91)

Algunos inspectores perciben que el "ABC padres" estaría comenzando a reemplazar a los modelos colegiados, intersectoriales y territoriales de planeamiento y gestión de la política, en la medida en que las UEGD comienzan a convocarse de modo intermitente en el distrito:

- Pero ellos con eso [el programa ABC padres] no solamente que se conforman, con eso ellos dicen que están cumpliendo con la ley. Por eso eliminan la UEGD (...) la UEGD está totalmente desbaratada. Acá en Hurlingham se convocó antes de que se fuera el anterior jefe. En el mes de marzo. Esa fue la última UEGD.

- ¿Y ya no va a volver a suceder...?

- No. No porque no la autorizan a la Jefa Distrital a realizar reuniones de UEGD. Los Sindicatos protestan: han hecho presentaciones... (Inspectora de nivel secundario, gestión Cambiemos)

\footnotetext{
${ }^{3}$ Fuente: portal www.abc.gov.ar, consultado en septiembre de 2017
} 
REVISTA DE LA ESCUELA DE CIENCIAS DE LA EdUCACIÓN, AÑO 17, NRO. 16, VOL. 2, JULIO A DICIEMBRE dE 2021. PÁGINAS 57-67. ISSN 2362-3349 (EN LínEA). RESISTIR DESDE EL ESTADO. LA CONSTRUCCIÓN DE ESTRATEGIAS "DESDE ABAJO" FRENTE A LOS MODOS DE GESTIÓN DE LAS POLITICAS HACIA LA ESCUELA SECUNDARIA OBLIGATORIA DE CAMBIEMOS. MAIDA DIYARIAN. INÉS RODRIGUEZ MOYANO.

Aunque desde lo formal las UEGD siguen vigentes, las modificaciones planteadas en la nueva regulación del año 2018 respecto a su constitución, objetivos y funcionamiento (Resolución de firma conjunto Nro. RESFC-2018-2016-GDEBA-DGCYE) introdujeron nuevos requerimientos que, según algunos inspectores entrevistados, muchas veces tornan inviable su realización. En la medida en que se extreman los criterios requeridos para la apertura de estos espacios, se refuerzan las lógicas burocráticas en detrimento de los aspectos sustantivos vinculados a la resolución intersectorial de problemas situados.

Sí, [antes] convocabas la UEGD en función a lo que ibas a tratar, a las necesidades. Ahora no, para celebrar una reunión UEGD necesariamente tenés que convocar a determinados referentes de la comunidad y llevarlo adelante. Y si algún porcentaje no está presente, no la podés desarrollar. Está todo como más rígido (Inspector de nivel secundario, gestión kirchnerista y Cambiemos).

Otras huellas del proceso de fragmentación y debilitamiento de las instancias de poder intermedio y local pueden también visualizarse en la inestabilidad de los cargos y la alta rotación de funcionarios que ocupan los puestos políticos de los niveles intermedios de la gestión respecto del periodo anterior. Esta dinámica es vista por varios de los inspectores como otro factor que contribuye a debilitar los procesos de construcción de poder a nivel territorial, en la medida en que vuelve frágiles las alianzas y obtura toda posibilidad de elaboración de lineamientos de política de largo plazo. Como hemos evidenciado en el trabajo de campo, desde el inicio de la nueva gestión a fines del 2015 han pasado por la jefatura distrital tres funcionarios. Asimismo, como lo confirma la actual Referente Regional de Políticas Socioeducativas, a la rotación de funcionarios en el cargo que ella ocupa se sumó la vacancia de una coordinación efectiva durante casi un año. La mentada complejidad del territorio, la falta de equipos, así como la dificultad de la nueva gestión de trabajar con las burocracias intermedias que venían de gestiones anteriores, parecen ser algunos de los motivos promotores de problemas de gobernabilidad del área en el territorio ${ }^{4}$ :

Hacía un rato que no había un coordinador de política socioeducativa en la región. Cuando yo tomo el cargo, hacía un año casi... en realidad, no es que hacía un año que no se cubría el puesto, sino que ningún coordinador regional se había centralizado en estar un tiempo prudencial para poder coordinar la gestión (...) Se iban. Se iban porque había cosas que eran bastante insostenibles en lo que es el trabajo de territorio, que es muy demandante, como que era demasiada la presión y la carga y no aguantaban y dejaban el trabajo. La persona más antigua que está ahora en la gestión (...) fue un poco el regional sin tener el cargo, y se ocupó de lo que es el territorio. (...) Y había mucha gente trabajando, que nosotros no hicimos la selección de ellos, venían de gestiones anteriores. Entonces la verdad es que la grieta está en todos lados, y al entrar nosotros, no sabían quién es Víctor, quién es Soledad, o quién soy yo. Se complejizaron las cosas, hubo falta de entendimiento, nosotros pretendimos alcanzar otro nivel de trabajo distinto (Referente Regional de Políticas Socioeducativas, gestión Cambiemos).

Como mencionábamos anteriormente, el gobierno de Cambiemos introduce nuevos programas que refuerzan lógicas de participación individual en detrimento de las organizaciones colectivas y las intervenciones intersectoriales. A su vez, las formas de participación promovidas tienden a desplazar ciertas responsabilidades por el cumplimiento del derecho a la educación desde el Estado a las escuelas. Esto se advierte en las nuevas formas de planificación y gestiones de la política socioeducativa introducidas, orientadas a transferir a los directores de las escuelas la responsabilidad de la toma de decisiones sobre la participación de sus instituciones en los programas. Basados en el diagnóstico de que los programas socioeducativos generaban el rechazo de los directivos al ser impuestos "desde arriba", el "cambio de perfil" del área iniciado con la actual gestión, adopta en febrero del 2018 la estrategia de "bajar" todos los programas vigentes delegando la responsabilidad de la continuidad de los mismos en los coordinadores de los programas y en su capacidad para convocar a los directivos a que presenten proyectos "innovadores" atendiendo a las particularidades de su entorno:

Lo que se modificó me parece que es lo más distintivo, que es el programa en sí. Si bien por qué sigue siendo el mismo, el espíritu del programa sigue siendo el mismo, cambiaron muchos ejes que facilitan que el programa sea más valorado por la escuela. ¿Qué pasaba antes? Vos tenías una escuela en donde se abría un Patio, o tenías una escuela en donde se ponía un CAl; un CAI es un Centro de Actividades Infantiles, un CAJ es un Centro de Actividades Juveniles. Y después tenías Patios Abiertos. En ese momento eran los tres programas bueno, Coros y Orquestas, Orquestas del Bicentenario. Pero digo, como para poder empezar a divisarte bien cada programa. Lo que sucedía es que el director tenía ese programa, y ese programa no había sido elegido por él, era una implementación. Y los directores viste que tienen ese miramiento como que "las escuelas son nuestras" "me están ocupando la escuela el sábado". Entonces era una rispidez constante entre el directivo y el coordinador, todo el tiempo era un problema porque el director

\footnotetext{
${ }^{4} \mathrm{~A}$ pesar de que la actual referente regional hace un año ocupa el cargo, llama la atención el desconocimiento sobre el lugar que ocupa el área en la actual estructura de gestión de la dirección, tanto a nivel provincial como a nivel regional. En efecto, convocó a la entrevista al referente que ocupó de hecho el cargo mientras había quedado vacante, para que pudiese aportar más información.
} 


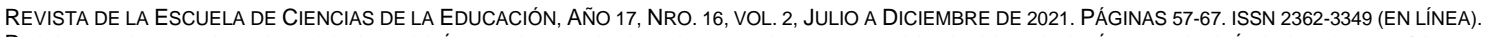
RESISTIR DESDE EL ESTADO. LA CONSTRUCCIÓN DE ESTRATEGIAS "DESDE ABAJO” FRENTE A LOS MODOS DE GESTIÓN DE LAS POLITICAS HACIA LA ESCUELA SECUNDARIA OBLIGATORIA DE CAMBIEMOS. MAIDA DIYARIAN. INÉS RODRIGUEZ MOYANO.

venía y reclamaba que le dejaron una sillita afuera, que le dejaron sucios los baños, etc. Y tenías escuelas que no, que valoraron mucho el programa, y otras que no lo querían. Entonces eso generaba una rispidez constante. Cuando cambia la directora provincial [de Políticas Socioeducativas], aprovechó e hizo un cambio muy genuino. La verdad que fue muy valiente, porque era un cambio radical en cuanto a que bajaba todos los proyectos. O sea, vos pensá que vos tenías, por decirte cualquier cosa, en San Martín, 15 programas. Estos programas se bajaban, o sea había programas que estaban hacía años, te estoy hablando de CAl y CAJ solamente, primero empezó el foco ahí. Caían, y el coordinador, que estaba en ese CAl, en ese CAJ, que estaba interesado en continuar su labor, tenía que ir a hablar con el directivo y desde el directivo proponer un proyecto innovador, que fue para mí mucho más significativo, porque tenía que ver con que el director era partícipe de ese proyecto, quería ese programa en su escuela y además sabía cuál era la realidad educativa de la comunidad (Referente Regional de Políticas Socioeducativas, gestión Cambiemos).

Según la voz de los inspectores distritales y de nivel, en Hurlingham dicha estrategia redundó en una baja significativa de las líneas socioeducativos que hasta el 2015 recibían todas las escuelas ${ }^{5}$. Al momento del trabajo de campo, según la información brindada por la inspección del nivel, de las 25 escuelas del distrito sólo funcionan tres CAJ y una sola institución bajo el programa de Escuelas Solidarias. Por otro lado, la eliminación de la figura del referente pedagógico de los programas que en el período previo articulaba con los inspectores del nivel, ha debilitado la dimensión pedagógica de las líneas de acción dejando al referente contable como instancia única de la gestión.

(...) El lugar de los referentes de políticas socioeducativas en los territorios, es contable. Nada más. Antes había un referente contable y había un referente pedagógico.

- ¿Y ahora el rol del referente pedagógico no existe?

- No existe más. Somos nosotros. Es directamente: el director gestiona el proyecto de esa línea, con los recursos que... y nosotros los supervisores, supervisamos. Acompañamos. Pero digamos, no hay una línea... no hay una línea, ¿me entendés? (Inspectora de nivel secundario, gestión Cambiemos).

En suma, como relata la Inspectora del distrito, las únicas líneas de acción para garantizar el derecho a la educación secundaria en Hurlingham a mediados del 2018 refieren a los ejes transversales comunes a demás niveles (prácticas del lenguaje, matemáticas, el egreso con terminalidad, y cuestiones que tienen que ver con el aspecto edilicio) que más que acciones concretas de política pública marcan lineamientos generales de trabajo sin brindar recursos ni programas específicos para intervenir sobre las problemáticas a nivel de las instituciones:

- Es que las políticas de acción, atraviesan todos los niveles. Estas cuatro que son prácticas del lenguaje; matemáticas; el egreso con terminalidad; y cuestiones que tienen que ver con el aspecto edilicio.

- Son transversales.

- Estos 4 ejes son los que conforman la política educativa para cada uno de los niveles. - Pero son temas? No es que hay recursos...

- Son ejes de trabajo. Luego lo que se hace es contextualizarlo distritalmente, y se contextualizan luego en cada nivel y cada modalidad, y se re contextualizan luego en cada uno de los servicios, desde la práctica del lenguaje: cómo lo voy a trabajar en primer año, en segundo. Cómo lo voy a hacer en matemática; el egreso; la certificación; el egreso con título. Entonces cada uno contextualiza de sus niveles y modalidades que tiene.

- Por ejemplo, antes, (...) no sé en qué estado está, si está funcionando el PMI. El Plan de Mejora Institucional que funcionaba en muchas escuelas y que usualmente lo utilizaban para gestionar espacios de apoyo y demás, y que ayudaba esto a promover la titulación de los estudiantes. Por eso te preguntamos concretamente. En términos de programa. Si, la preocupación está por la terminalidad: ahora, ¿con qué recursos cuentan las escuelas para empujar hacia eso? Te traigo el PMI porque en su momento, muchas escuelas lo usaban para eso. Ahora creo que el PMI no estaría...

- No, no está funcionando. Con las estrategias institucionales y con los recursos con que cuenta la escuela. Hoy por hoy, subsidios han llegado a través del programa SITRARED, en donde está direccionado hacia las instituciones.

- Esto es socioeducativo? ¿Lo socioeducativo está vinculado a eso?

- Si, lo socioeducativo. Pero no todas las escuelas están siendo beneficiadas hoy por hoy. En un momento, todas las escuelas recibieron SITRARED, por lo cual había un dinero para gastar e invertir en cada una de las instituciones con lo que tenía que ver con equipamiento tecnológico o recursos didácticos. Hoy por hoy son muy pocas las escuelas que tienen habilitado el dinero para

\footnotetext{
${ }^{5}$ Las líneas discontinuadas señaladas son: de prevención del abandono; de equipamiento educativo, de orquestas juveniles, de fortalecimiento de la Educación Física y de fortalecimiento de la Educación Artística.
} 


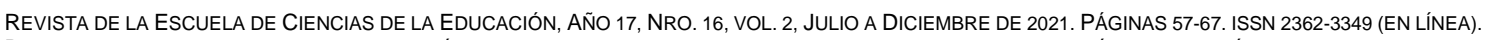
RESISTIR DESDE EL ESTADO. LA CONSTRUCCIÓN DE ESTRATEGIAS “DESDE ABAJO” FRENTE A LOS MODOS DE GESTIÓN DE LAS POLITICAS HACIA LA ESCUELA SECUNDARIA OBLIGATORIA DE CAMBIEMOS. MAIDA DIYARIAN. INÉS RODRIGUEZ MOYANO.

poder incrementar los recursos didácticos en las escuelas (Inspectora distrital, gestión Cambiemos).

\section{Las dinámicas de "resistencia" y la construcción de las políticas para el nivel secundario "desde abajo"}

A la par que desde el nivel provincial se promueven diferentes mecanismos tendientes a concentrar el poder en los niveles centrales de gobierno y debilitar y fragmentar las instancias de poder intermedio y local, al poner el foco en la escala territorial es posible observar procesos que imprimen matices a aquellas orientaciones. En efecto, frente al avance de nuevas políticas educativas definidas por el gobierno provincial y nacional que refuerzan la lógica compensatoria, meritocrática y tecnocrática, los equipos de inspección del nivel secundario del distrito desarrollan verdaderas estrategias de "resistencia", no sólo recontextualizando las orientaciones diseñadas "desde arriba" sino también construyendo "desde abajo" políticas dirigidas al nivel secundario. Así, ante la insuficiencia de recursos y políticas públicas para garantizar el derecho a la educación en el nivel secundario, en el distrito se vislumbra la emergencia de nuevas alianzas territoriales que tensionan las dinámicas de atomización y fragmentación del sistema. Se trata pues de prácticas que configuran una realidad alternativa a la planteada desde el nivel central, en fuerte articulación con distintos actores del territorio, entre ellos la Universidad local y otras áreas de gobierno municipal. La idea de "resistencia" como posibles acciones que se distancian de los lineamientos centrales aparece en el discurso de los Inspectores del nivel:

Digo, lo que pasa en, lo que sigue pasando en las comunidades, en el quehacer de las comunidades, no tiene nada que ver con lo que plantea la Dirección General de Educación. Son dos mundos, dos realidades completamente distintas. Por un lado, tenemos la vida de las escuelas, la de las comunidades... bueno por eso podemos resistir. Nosotros estamos resistiendo. (Inspectora de nivel secundario, gestión Cambiemos)

Para comprender estos relativos márgenes de autonomía de los Inspectores de Enseñanza es necesario tener en cuenta que, a diferencia de los Inspectores Regionales y Distritales, dichos actores son funcionarios de carrera que acceden a sus cargos por concursos públicos, de modo que formalmente su posición se ampara en un conocimiento técnico que excede a las gestiones políticas de gobierno. En el caso del distrito de Hurlingham, la llegada de Cambiemos coincidió con la renovación de los cargos de Inspección del nivel que serán ocupados por actores con trayectorias profesionales de marcada impronta militante y experiencia de trabajo territorial. Esta modalidad de reclutamiento propia de las burocracias modernas, junto a la trayectoria profesional y las identidades políticas de los inspectores, seguramente estén incidiendo en las posibilidades de construcción de otras alianzas en el territorio y habiliten posiciones que se distancian de las directrices y orientaciones emanadas desde las instancias superiores de la estructura de gobierno. Esta capacidad de "resistencia" ligada a las modalidades de acceso al cargo y a la trayectoria de quien lo ocupa es reconocida por los propios inspectores:

Estamos en plena tracción entre lo que se pide desde el nivel central y con la formación, una filosofía y una ética que uno tiene en relación a lo que significa ser supervisor (Inspectora de nivel secundario, gestión Cambiemos).

- (...) por eso no nos quieren a nosotros, porque nosotros somos de carrera ¡Los supervisores somos de carrera! (...) Tienen que cambiar todo, la ley, o sea... no tienen forma de sacarnos. Por eso, bueno, el derrotero del control, el menosprecio, la subestimación, el maltrato. Todo esto está hecho a propósito, para que nos vayamos (Inspectora de nivel secundario, gestión Cambiemos).

El proyecto Trayectorias Educativas Protegidas desarrollado en el distrito desde el año 2017 puede leerse bajo esta clave. Se trata de una iniciativa propuesta por el propio equipo de inspección de educación secundaria destinada a proteger las trayectorias de los estudiantes de sectores de mayor vulnerabilidad social bajo estrategias que buscan distanciarse de la "pedagogía del mérito" (Araya y Martinez, 2020). Sus líneas de acción incluyen el desarrollo de capacitaciones para directivos y la elaboración de proyectos institucionales que incorporan propuestas de flexibilización del régimen académico.

El dispositivo, que involucra a las 25 escuelas secundarias orientadas y a los 3 establecimientos técnicos del distrito, fue gestado e implementado en articulación con otras instituciones del territorio tales como la Universidad Nacional, el Servicio Local de Promoción y Protección de Derechos, la Secretaría de Salud, la Dirección de Niñez, el Centro de Articulación Penal para Jóvenes, la Dirección de Género, la Comisaría de la Mujer, entre otras. De este modo, la red intersectorial del municipio, que según los inspectores del nivel ha sido revitalizada desde la asunción del intendente kirchnerista Zabaleta en el año 2015, ha jugado un papel clave en el desarrollo de acciones dirigidas al nivel, incluso para traccionar la conformación de las escuelas secundarias que había quedado inconclusa en la gestión previa. Apoyándose en estas alianzas con los organismos municipales conducidos políticamente por el gobierno distrital kirchnerista, el equipo de inspección logra articular acciones e iniciativas que buscan distanciarse de las orientaciones de la política educativa de Cambiemos. En este sentido, la Mesa Distrital de Inclusión 


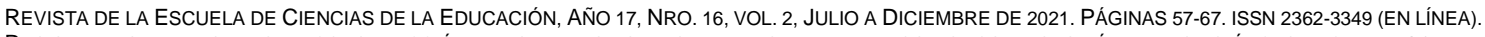
RESISTIR DESDE EL ESTADO. LA CONSTRUCCIÓN DE ESTRATEGIAS "DESDE ABAJO” FRENTE A LOS MODOS DE GESTIÓN DE LAS POLITICAS HACIA LA ESCUELA SECUNDARIA OBLIGATORIA DE CAMBIEMOS. MAIDA DIYARIAN. INÉS RODRIGUEZ MOYANO.

Educativa ${ }^{6}$ que reúne en forma intersectorial a distintas áreas estatales se configura bajo la gestión de Cambiemos como un ámbito importante de discusión y construcción de políticas para el nivel, desde donde se recoge información relevante para poder intervenir sobre problemáticas situadas del distrito. Es en dicho espacio donde se habilitó la articulación del equipo de inspección de nivel secundario con el Servicio Local de Niñez ${ }^{7}$ y otras áreas de gobierno, dando pie a la creación del proyecto piloto sobre Trayectorias Educativas Protegidas. Las propias inspectoras señalan la relevancia de aquellas articulaciones y del espacio intersectorial de trabajo:

- A su vez, articulamos con los organismos municipales, por ejemplo, la secretaría de género, el servicio local de niñez... según las problemáticas...

- ¿Y cómo es esa articulación? Contanos un ejemplo.

- Hay una Mesa de Inclusión donde se trabajan todos los casos de las problemáticas que implican...

- ¿Se reúnen cada tanto tiempo?

- Se reúne mensualmente, y ahí convergen todos. (...) El servicio local de niñez detectó junto con los datos, los relevamientos, eh... en muchos casos a nosotros nos sirven de insumos, que muchos desconocemos.... Pero por ejemplo tenemos 500 pibes y pibas que no están en la escuela. (...) de los cuales, 170 están judicializados. (...) Entonces, que esos pibes se reincorporen, es un desafío. Entonces, ¿cómo garantizamos nosotros que esos pibes y pibas se reincorporen? Trabajando fuertemente con la profesionalización de los directores, de los equipos de conducción, para que una vez que sean acercados los pibes a las escuelas, se implemente un dispositivo de trayectorias protegidas. Y esto es lo que vamos a presentar nosotros: es un dispositivo para seguir las trayectorias escolares....

- ¿Con "nosotras" a quiénes te referís?

- Al equipo de supervisión de educación secundaria de Hurlingham. (...) Bueno, nosotros pensamos, (...) a partir de los niveles de ausentismo, de abandono y de repitencia, la escuela tiene que... si bien tiene toda la normativa que habla de inclusión y obligatoriedad, todavía no había un dispositivo que obligara a la escuela a que cumpliera con ese objetivo. Entonces ahí pensamos en este dispositivo que se llama "Trayectorias Escolares Protegidas". (...) Se armó este año un dispositivo de profesionalización de los directores donde trabajamos autoridad, convivencia... un montón de temas que atraviesan y que los directores necesitan más instrumentos, más bibliografía, un marco que sustente y que avale las decisiones que se pueden tomar.

- Pero es un proyecto que surgió de Ustedes.

- De nosotros. (Inspectora de nivel secundario, gestión Cambiemos)

En eso [sobre el problema de abordar las trayectorias] nos seguimos manejando con las mismas líneas que teníamos con Claudia [Bracchi, directora provincial de Educación Secundaria de la gestión kirchnerista], sólo que sin los recursos.

- ¿Y cómo lo hacen?

- Y... lo hacemos... por suerte contamos con los CIEs [Centros de Investigación Educativa], están Ustedes. (...) Yo siempre les digo a los profesores (...) cada vez que iniciamos las jornadas de capacitación, que aprovechen esta instancia porque no todos los distritos tienen la posibilidad de tener una Universidad codo a codo.

- O sea que (...) ¿quiénes son tus aliados en esta tarea?

- El municipio... la Universidad...mis compañeras, el CIE, el Servicio Local de Promoción y Protección de Derechos, las organizaciones barriales, los sindicatos. Los sindicatos... (...) En este momento de la Dirección General de Educación no hay nada... más que control. No hay nada más que control. (Inspectora de nivel secundario, gestión Cambiemos)

Según los inspectores entrevistados, el proyecto sobre Trayectorias Educativas Protegidas busca proponerse como una alternativa frente al desfinanciamiento y la ausencia de políticas universales dirigidas al nivel secundario. Si bien no cuentan con un financiamiento especial para su implementación y se apoyan en recursos del municipio como la universidad local, los actores destacan que la propuesta se enmarca en la normativa vigente que alienta la promoción de los medios necesarios para garantizar la inclusión y la continuidad educativa:

- (...) este proyecto de "Trayectorias Escolares Protegidas", todas estas medidas que están tratando de llevar adelante, ¿con qué recursos lo implementan? Porque no es una política que te venga desde la inspección. Pero ¿qué instrumentos, qué recursos, ¿cómo hacen para...?

\footnotetext{
${ }^{6} \mathrm{Si}$ bien las Mesas Distritales de Inclusión Educativa se crearon en 2009 en el marco de la implementación de la Asignación Universal por Hijo, en Hurlingham, lograron institucionalizarse recién en 2018 bajo la gestión de Cambiemos, nueve años después de su creación indicando una vez más la existencia de mediaciones entre las definiciones propuestas en el diseño de las políticas desde el nivel central y sus modos de apropiación local.

${ }^{7}$.Dicha articulación permitió al equipo de inspección contar con estadística certera sobre la cantidad de jóvenes que estaban fuera del sistema.
} 


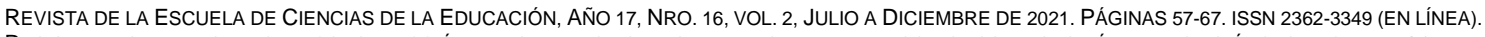
RESISTIR DESDE EL ESTADO. LA CONSTRUCCIÓN DE ESTRATEGIAS “DESDE ABAJO” FRENTE A LOS MODOS DE GESTIÓN DE LAS POLITICAS HACIA LA ESCUELA SECUNDARIA OBLIGATORIA DE CAMBIEMOS. MAIDA DIYARIAN. INÉS RODRIGUEZ MOYANO.

- Con la fuerza de la ley. ¿Qué otro recurso hay más que la ley? O sea, nosotros socializamos la normativa (Inspectora de nivel secundario, gestión Cambiemos).

La vigencia de la Ley de Educación Nacional Nro. 26.206, de la Ley de Educación Provincial Nro. 13.688 y de acuerdos marco para la educación secundaria definidos en el Consejo Federal de Educación ${ }^{8}$. fundados en la perspectiva del derecho social a la educación, opera como garantía para el desarrollo de acciones que desde la mirada de los actores profundizan los procesos de democratización social y educativa.

\section{A modo de cierre}

El estudio de las modalidades de gestión de las políticas dirigidas a garantizar la obligatoriedad del nivel secundario a escala local nos permitió reparar en los matices que los procesos locales de implementación les imprimen a las orientaciones políticas definidas desde los niveles centrales de gobierno. Sólo considerando a las políticas como resultado de relaciones de fuerza entre los múltiples actores que intervienen en su diseño e implementación, es posible comprender las distancias y resignificaciones que se producen en aquella trayectoria. La identificación de la multiplicidad de actores, escenarios, lógicas y temporalidades específicas que incluso pueden trascender a las gestiones de gobierno, interpela la supuesta convergencia automática entre las normas, la institucionalidad y las prácticas concretas y es esta complejidad, que se expresa en el territorio en tanto unidad de implementación de las políticas, la que nos interesó captar.

En este sentido, si bien como hemos mostrado en este artículo las políticas educativas del gobierno de Cambiemos se han caracterizado por el despliegue de mecanismos tendientes a debilitar las instancias de poder intermedio y local en el marco de una agenda pública en la que la dimensión pedagógica como eje estructurador en la definición de las políticas educativas pierde centralidad, a nivel territorial emergen estrategias de resistencias que ponen en tensión algunas orientaciones de la política educativa definidas desde los niveles centrales.

El proyecto de Trayectorias Educativas Protegidas propuesto por las inspectoras de nivel en articulación con organismos locales fue acogido por la Inspección Distrital y Regional convirtiéndose, según los propios actores, en la principal política educativa dirigida a la educación secundaria implementada en el distrito frente a la ausencia de otros programas.

Es posible advertir entonces que las políticas efectivamente implementadas en el territorio han recorrido un camino en dirección de "abajo" hacia "arriba", logrando poner en tensión algunos sentidos de la política educativa de Cambiemos definida desde los niveles centrales. Considerando esta capacidad de agencia de los actores locales sostenemos que, más allá de las orientaciones generales de la agenda asociadas a un gobierno de signo político determinado, el análisis de la trayectoria de las políticas requiere a su vez reparar en las estrategias informales (no institucionales) desplegadas por las burocracias intermedias y en las alianzas movilizadas en ese plano con actores que inciden en la escala territorial y que trascienden las fronteras de la gestión educativa.

\section{Referencias bibliográficas}

Agoff, S.; Bertranou J, y Foglia C. (2012). "Las políticas educativas y la reconstrucción de la gobernabilidad de la educación en la provincia de Buenos Aires”. Ponencia presentada en el IX Seminario de la Red Estrado "Políticas educativas en América Latina. Praxis docente y transformación social", Universidad de Chile, del 18, 19 y 20 de julio de 2012, Santiago de Chile.

Araya, S. y Martinez, E. (2020). Se requiere mayor financiamiento. Serie La educación en debate, Nro. 79, Abril 2020, 3 , UNIPE.

Ball, S. (2002). Textos, discursos y trayectorias de la política: la teoría estratégica. Escuela de educación, UNC, Córdoba.

Canelo, P. y Castellani, A. (2016). Perfil sociológico de los miembros del gabinete inicial del presidente Mauricio Macri, Observatorio de las elites, Informe de Investigación No1. IDAES, UNSAM.

Feldfeber, M. (2011). ¿Quién garantiza el derecho a la educación en un país federal? En Dacunha, C., Vieira De Sousa, J. y Da Silva, M. (organizadores). Políticas Públicas de Educação na América Latina: lições aprendidas e desafios, Faculdade de Educação da Universidade de Brasilia, Autores Associados. Campinas.

Giovine, R. (2012). El arte de gobernar el sistema educativo. Discursos de Estado y redes de integración socioeducativas. Bernal: Universidad Nacional de Quilmes.

Natanson, J. (2018). ¿Por qué? La rápida agonía de la Argentina kirchnerista y la brutal eficacia de una nueva derecha. Siglo XXI Editores, Buenos Aires.

Paura, V. y Zibecchi, C. (2013). Políticas sociales y territorio: lógicas institucionales y lógicas de los actores. Abordajes, hipótesis y debates vigentes. Ponencia presentada en el XXIX Congreso ALAS: "Crisis y emergencia sociales en América Latina", Santiago de Chile, del 30 de septiembre al 4 de octubre de 2013.

Rodríguez Moyano, I. y Diyarian, M. (2020). Recentralización del poder y debilitamiento de la participación. Una aproximación a los modos de construcción de las políticas hacia la escuela secundaria obligatoria en la provincia de buenos Aires a escala territorial (2015-2020), en Revista Sociedad de la Facultad de Ciencias Sociales de la Universidad de Buenos Aires, Num. 40, 174-190 ISSN: 2618-3137. Disponible en: https://publicaciones.sociales.uba.ar/index.php/revistasociedad/index

Suasnábar, C. et. al. (2017). La gestión educativa de la Alianza Cambiemos en la provincia de Buenos Aires: una exploración de las trayectorias profesionales, identidades políticas y la agenda de política. Ponencia

\footnotetext{
${ }^{8}$ Nos referimos, entre otras, a las resoluciones del Consejo Federal de Educación № 84/2009, 93/2009 y 103/2010.
} 
REVISTA dE LA ESCUELA de CIENCIAS DE LA EduCACIÓN, AÑO 17, NRO. 16, VOL. 2, JULIO A DiCIEMBRE dE 2021. PÁGINAS 57-67. ISSN 2362-3349 (EN LíNEA). RESISTIR DESDE EL ESTADO. LA CONSTRUCCIÓN DE ESTRATEGIAS "DESDE ABAJO” FRENTE A LOS MODOS DE GESTIÓN DE LAS POLITICAS HACIA LA ESCUELA SECUNDARIA OBLIGATORIA DE CAMBIEMOS. MAIDA DIYARIAN. INÉS RODRIGUEZ MOYANO.

presentada en el $9^{\circ}$ Congreso Latinoamericano de Ciencia Política, organizado por la Asociación Latinoamericana de Ciencia Política (ALACIP), Montevideo, 26 al 28 de julio de 2017.

Virilio, P. (1989). La máquina de visión. Madrid: Cátedra. 